\title{
Metabolomic analysis and biochemical changes in the urine and serum of streptozotocin-induced normal- and obese-diabetic rats
}

\begin{abstract}
ABSTRCT
Diabetes mellitus (DM) is a chronic disease that can affect metabolism of glucose and other metabolites. In this study, the normal-and obese-diabetic rats were compared to understand the diabetes disorders of type 1 and 2 diabetes mellitus. This was done byevaluating their urine metabolites using proton nuclear magnetic resonance (1H NMR)-based metabolomics and comparing withcontrols at different time points, considering the induction periods of obesity and diabetes. The biochemical parameters of theserum were also investigated. The obese-diabetic model was developed by feeding the rats a high-fat diet and inducing diabeticconditions with a low dose of streptozotocin (STZ) $(25 \mathrm{mg} / \mathrm{kg} \mathrm{bw})$. However, the normal rats were induced by a high dose of $S T Z(55 \mathrm{mg} / \mathrm{kg}$ bw $)$. A partial least squares discriminant analysis (PLS-DA) model showed the biomarkers of both DM typescompared to control. The synthesis and degradation of ketone bodies, tricarboxylic (TCA) cycles, and amino acid pathwayswere the ones most involved in the variation with the highest impact. The diabetic groups also exhibited a noticeable increase inthe plasma glucose level and lipid profile disorders compared to the control. There was also an increase in the plasma cholesteroland low-density lipoprotein (LDL) levels and a decline in the high-density lipoprotein (HDL) of diabetic rats. The normal-diabetic rats exhibited the highest effect of all parameters compared to the obese-diabetic rats in the advancement of the DMperiod. This finding can build a platform to understand the metabolic and biochemical complications of both types of DM and cangenerate ideas for finding targeted drugs.
\end{abstract}

Keyword: 'H NMR-based metabolomics; Diabetes mellitus; Multivariate data analysis; Metabolic pathway. 